\title{
Unraveling Mechanisms that Control Platelet Production
}

\author{
Joseph E. Italiano, Jr., $\mathrm{PhD}^{1}$ \\ ${ }^{1}$ Division of Hematology, Brigham and Women's Hospital, Boston \\ Children's Hospital, Harvard Medical School, Boston, Massachusetts \\ Semin Thromb Hemost 2013;39:15-24.

\begin{abstract}
Address for correspondence and reprint requests Joseph E. Italiano, Jr., PhD, Division of Hematology, Brigham and Women's Hospital, Karp Bldg, 5th floor, Room 216, Boston, MA 02115 (e-mail: jitaliano@rics.bwh.harvard.edu).
\end{abstract}
Abstract Keywords
- megakaryocyte
- platelet
- proplatelet
- thrombopoiesis
- cytoskeleton

Platelets are formed by giant precursor cells called megakaryocytes that reside within the bone marrow. The generation of platelets, and their release into the bloodstream by megakaryocytes, requires a complex series of remodeling events powered by the cytoskeleton to result in the release of many platelets from a single megakaryocyte. Abnormalities in this process can result in thrombocytopenia (low platelet count) and can lead to increased risk of bleeding. This review describes the process of platelet production in detail and discusses new insights into novel platelet biology.
Blood platelets, tiny cells shed by megakaryocytes, circulate throughout blood vessels and survey the integrity of the vascular system. Each day an adult produces about 100 billion platelets. Although our understanding of platelet production has grown considerably in recent years, the mechanisms by which platelets are produced from megakaryocytes largely remains to be defined. The development of megakaryocyte cultures that produce bona fide platelets has provided a means to study the intermediate structures called "proplatelets," long, thin extensions of the megakaryocyte cytoplasm that function as assembly lines for platelet production. This article represents an integrated review on the mechanisms of platelet production. Because the cytoskeleton provides the force to power the production of platelets, this review first considers the cytoskeletal elements that provide the structure of the resting platelet cytoskeleton. The review then focuses on the mechanisms that lead to the elaboration of proplatelets and release of individual platelets. Also discussed are the new and emerging roles of platelets that extend beyond thrombosis and hemostasis.

\section{Structure and Cytoskeleton of Circulating Platelets}

Megakaryocyte development culminates with the release of mature discoid platelets with average dimensions of $3.0 \times 5.0 \mu \mathrm{m}$. The plasma membrane of the resting platelet is replete with transmembrane receptors. As much as $20 \%$ of the surface area is occupied with receptors, which include ligands for thrombin, IgG (FcrRIIA), and the major surface glycoproteins $\mathrm{GP}_{\mathrm{IIb}} \beta_{3}$ and GPIb/IX/V. The cytoplasm of platelets contains two types of secretory granules: dense- and $\alpha$ granules. The disc shape of the platelet is maintained by a very unique and specialized cytoskeleton, which functions as the molecular struts and girders of the cell. ${ }^{1}$ The main components of the platelet cytoskeleton are a marginal microtubule coiled repeatedly into a band, a spectrin-based membrane skeleton, and a three-dimensional network of cross-linked actin filaments.

The marginal band of resting platelets is located at the cell periphery and consists of multiple microtubules, coiled up 8 to 12 times. $^{2}$ Microtubules are long, hollow polymers formed from tubulin dimers that provide the force for movement in many cellular functions, including mitotic chromosome segregation as well as organelle translocation. Platelets and megakaryocytes contain primarily the $\beta-1$ isoform of tubulin. $\beta-1$ Tubulin is the most highly divergent $\beta$ tubulin isoform and is hematopoietic specific. Analysis of $\beta-1$ tubulin knockout mice points to a critical cellular function for $\beta-1$ tubulin in platelet biogenesis. These mice exhibit a bleeding phenotype, have reduced platelets, and contain defective microtubule coils. $^{3}$ Consistent with these observations, a mutation in the human $\beta-1$ tubulin gene has been identified, and analysis has revealed large spherical platelets with a microtubule coil that is highly disorganized. Platelets also contain the motor proteins cytoplasmic dynein and kinesin, which can bind to
Issue Theme Hot Topics IV; Guest Editor, Emmanuel J. Favaloro, PhD, FFSc (RCPA).
Copyright $\odot 2013$ by Thieme Medical Publishers, Inc., 333 Seventh Avenue, New York, NY 10001, USA. Tel: +1(212) 584-4662.
DOI http://dx.doi.org/ $10.1055 / \mathrm{s}-0032-1331157$. ISSN 0094-6176. 
microtubules and exert force. The generation of the microtubule coil is a critical event in the final stages of platelet production, and the mechanisms of its construction are discussed in more detail later in this review.

An elaborate network of spectrin and associated proteins laminates the underside and provides structural support for the plasma membrane of the resting platelet. ${ }^{1}$ The spectrin strands interact to form a hexagonal lattice and are densely decorated with attached membrane glycoprotein. The platelet spectrin network is structurally similar to the membrane skeleton of the erythrocyte, except its spectrin molecules interconnect to actin using the ends of long filaments instead of short oligomers of actin. Individual molecules of actin come together to form short oligomers that subsequently undergo assembly into long filaments. Actin is the most abundant of all proteins in platelets, making up about $20 \%$ of the total protein. The filamentous actin element of resting platelets is arranged into approximately 2,000 actin filaments that are interconnected, at various points, into a rigid network by the actin crosslinking protein filamin A (FLNa). ${ }^{1}$ FLNa subunits selfassociate in solution into long homodimers. ${ }^{4}$ FLNb is expressed in platelets at one-tenth the level of FLNa $(12,000$ copies/platelet). Andrews and Fox ${ }^{5}$ were the first to discover that FLNa links GPIb/IX/V (which forms the von Willebrand factor receptor on the platelet membrane) to actin filaments in the cytoplasm. The second rod domain of FLNa (repeat 17) has a binding site for the cytoplasmic tail of GPIb- $\alpha$ component of GPIb/IX/V, and cell biologic experiments have shown that most platelet FLNa ( $\geq 90 \%$ ) is in complex with GPIb- $\alpha .{ }^{4}$ Because a large percentage of FLNa is attached to actin, it positions the von Willebrand factor receptor on the surface of the platelet over the underlying linear filaments in the platelet cytoskeleton. Platelets from patients with BernardSoulier syndrome (who lack the von Willebrand factor receptor) also lack this connection, and their platelets are unusually large and fragile. As Bernard-Soulier syndrome patients have a severe bleeding diathesis and thrombocytopenia, this suggests that GPIb/IX/V is important for normal platelet generation. ${ }^{6}$ This possibility is further suggested by the low platelet count and unusual morphology of platelets observed in mice lacking the GPIb- $\alpha$ receptor. ${ }^{7}$ Although the FLNa-von Willebrand factor receptor connection has been characterized at length in platelets, the role of this molecular linkage in platelet production is not fully understood.

\section{Mechanisms of Platelet Production}

\section{Proplatelet Model of Platelet Production}

Megakaryocytes are highly specialized cells that expand and become polyploid through a process called endomitosis. As megakaryocytes develop, their cytoplasm increases in size and becomes full of platelet-specific granules; at the same time the megakaryocyte develops a highly invaginated demarcation membrane system. Megakaryocyte maturation comes to completion with the release of platelets into the bloodstream. Past studies focusing on the mechanics of this process have been hindered by the rarity of megakaryocytes in the bone marrow $(<0.1 \%$ of the total cells $)$ and the lack of in vitro systems that faithfully reconstitute platelet biogenesis. However, the discovery of thrombopoietin, the major cytokine that regulates the growth and development of megakaryocytes, and the emergence of cell culture systems that reconstitute bona fide platelet generation have resulted in substantial progress toward understanding the maturation of megakaryocytes. ${ }^{8}$

The currently favored model of platelet production recognizes that differentiated megakaryocytes extend long cytoplasmic processes, designated proplatelets. ${ }^{9}$ Proplatelets function as the assembly lines of platelet production and comprise platelet-sized swellings in tandem arrays that are linked by thin cytoplasmic bridges (-Fig. 1). ${ }^{10}$ Although extensive characterization of proplatelets remains incomplete, these processes have been recognized both in vitro and in vivo, and proplatelet-producing megakaryocytes generate platelets that are structurally and functionally similar to blood platelets. ${ }^{11}$ Proplatelets have been observed extending from megakaryocytes in the bone marrow through junctions in the lining of blood sinuses where they have been hypothesized to be released into the circulation and undergo additional fragmentation into platelets. ${ }^{9}$ Furthermore, mice

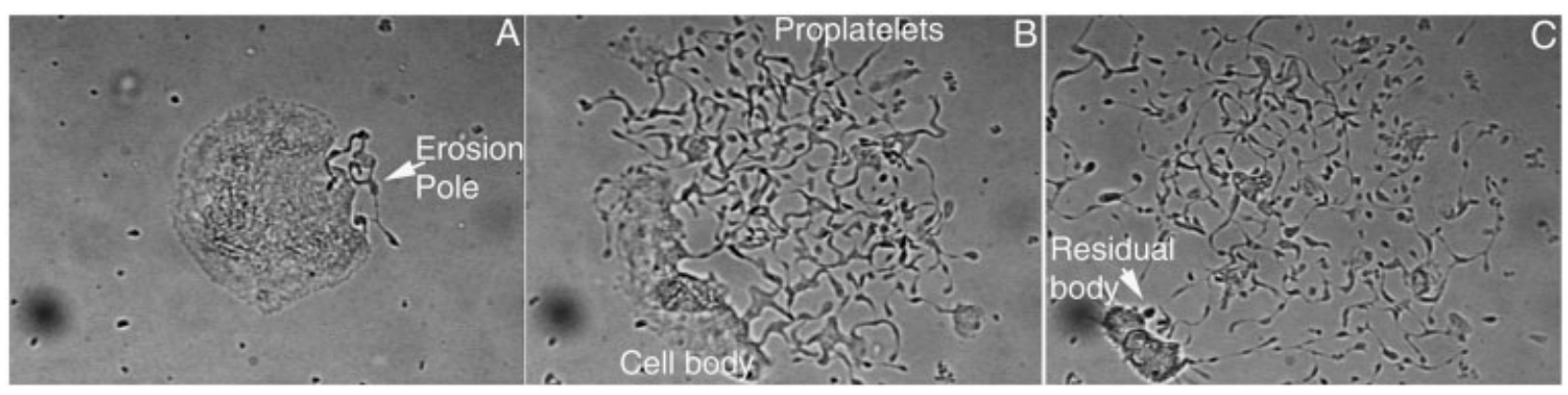

Fig. 1 Generation of proplatelets by a mouse megakaryocyte. Time-lapse sequence of a megakaryocyte, showing the essential events that lead to elaboration of proplatelets in vitro. (A) Platelet production starts when the megakaryocyte cytoplasm starts to erode at one pole (arrow). (B) The majority of the megakaryocyte cytoplasm has been converted into multiple proplatelet processes that continue to lengthen and form swellings along their length. These processes are highly dynamic and undergo bending and branching. (C) Once the bulk of the cytoplasm has been converted into proplatelets, the entire process ends in a rapid retraction that separates the released proplatelets from the residual cell body. (Adapted from: Italiano JE Jr., Lecine P, Shivdasani RA, Hartwig JH. Blood platelets are assembled principally at the ends of proplatelet processes produced by differentiated megakaryocytes. J Cell Biol 1999;147(6):1299-1312.) 
lacking distinct hematopoietic transcription factors, such as nuclear factor erythroid-derived 2, have severe thrombocytopenia and fail to produce proplatelets in vitro, highlighting the parallel to platelet genesis in vivo. ${ }^{12}$ Before proplateletbased models, platelets were thought to form in the cytoplasm of the megakaryocyte and undergo fragmentation along the boundaries of the demarcation membrane system. ${ }^{13}$ However, evidence now indicates the primary role of the demarcation membrane system is to serve as an extensive membrane reservoir that is required for proplatelet elaboration. ${ }^{14-16}$ The exact mechanism and the locations of platelet production are, however, still controversial. Production of platelets from megakaryocytes within capillary beds has been proposed as an alternative mechanism, but it is difficult to demonstrate in the mouse or other species. Proplatelet formation, originally identified within bone marrow sinusoids, may also take place partially in the bloodstream.

\section{Stages of Platelet Production}

The development of megakaryocyte cultures that faithfully reconstitute platelet formation has provided model systems to study megakaryocytes in the act of forming proplatelets. ${ }^{11,17}$ Live cell microscopy reveals both spatial and temporal changes leading to the generation of proplatelets (-Fig. 1). ${ }^{18}$ Remodeling of the megakaryocyte cytoplasm concentrates almost all the intracellular contents into proplatelet processes and their platelet-size particles, which, in the final stages, appear as beads linked by thin cytoplasmic bridges. The transformation unfolds over 3 to 10 hours and begins in a polarized fashion with the erosion of one side of the megakaryocyte cytoplasm. Thick pseudopodia-like processes initially form and then extend into thin tubes of uniform diameter of 2 to $4 \mu \mathrm{m}$. The thin tubules subsequently undergo a dynamic bending and branching process and develop periodic platelet-sized swellings that span their length (-Fig. 2). Ultimately, the megakaryocyte is converted into a residual naked nucleus surrounded by an elaborate network of proplatelets (-Fig. 1C). Megakaryocyte maturation ends when a fast retraction that separates the proplatelet fragments from the cell body releases the processes into culture. The subsequent rupture of the cytoplasmic bridges between platelet-size segments is thought to release individual platelets into the circulation. ${ }^{18}$

\section{Microtubules Power the Elongation of Proplatelets}

Some of the first insights into the cytoskeletal mechanisms that power platelet formation dates from the work of Tablin and Leven, who used microtubule inhibitors to establish that proplatelet extension is dependent on microtubules. ${ }^{19}$ The

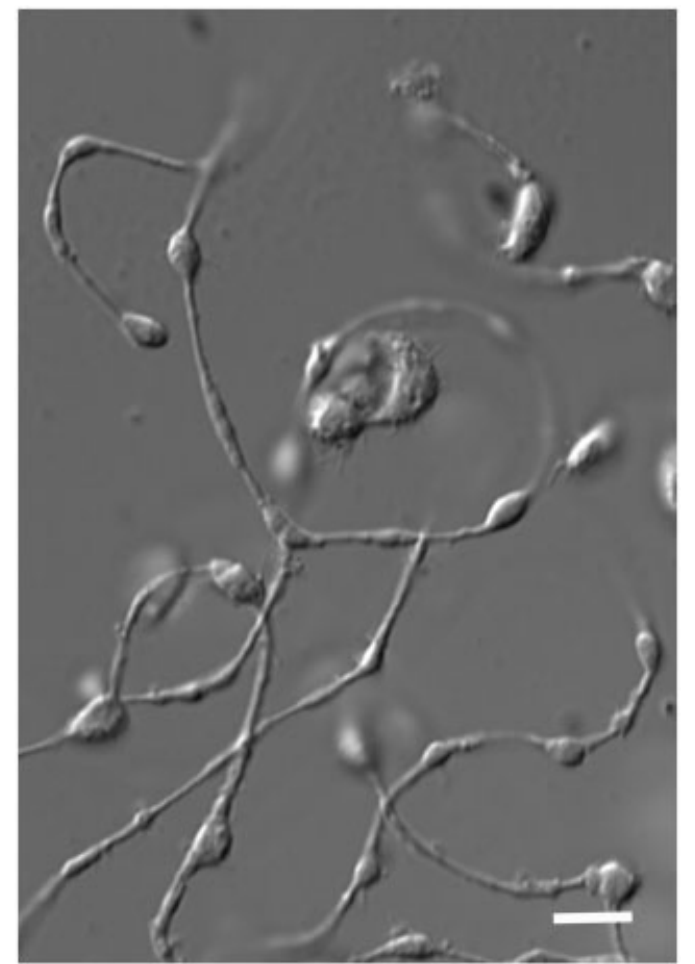

Fig. 2 Architecture of proplatelets. Image of a differential interference contrast micrograph of proplatelets extending from a mouse megakaryocyte culture. The hallmark features of proplatelets, including the tip, shaft, branch points, and platelet-sized swellings that decorate the length, are observed. Scale bar, $5 \mu \mathrm{m}$.

megakaryocyte cytoskeleton, which is composed of microtubules, actin, and spectrin, functions as the engine that powers the production of platelets ( - Table 1, - Fig. 3). Proplatelet formation and elongation is dependent on microtubule function, because treatment of megakaryocytes with drugs that disassemble microtubules, such as vincristine or nocodazole, blocks proplatelet formation. ${ }^{19}$ Examination of the microtubule cytoskeletons of proplatelet-producing megakaryocytes provides insights into how microtubules contribute to platelet production (-Fig. 4). The microtubule cytoskeleton in megakaryocytes undergoes a striking remodeling during proplatelet production. ${ }^{18}$ In immature, round megakaryocytes without proplatelets, microtubules extend out from the cell center (centrosome) to the cortex. As thick pseudopodial processes form during the early stage of proplatelet production, cortical microtubules combine into thick bundles located under the plasma membrane of these structures. When pseudopodia start to extend (at an average rate of about $0.85 \mu \mathrm{m} / \mathrm{min}$ ), microtubules form thick linear arrays that core the length of the proplatelets. The bundles of

Table 1 Cytoskeletal machinery of platelet production

\begin{tabular}{|l|l|}
\hline Cytoskeletal component & Major function(s) in platelet production \\
\hline Microtubules & Proplatelet elongation \\
\hline Actin & Proplatelet bending and branching \\
\hline Spectrin & Formation of the invaginated membrane system and stabilization of proplatelet architecture. \\
\hline
\end{tabular}




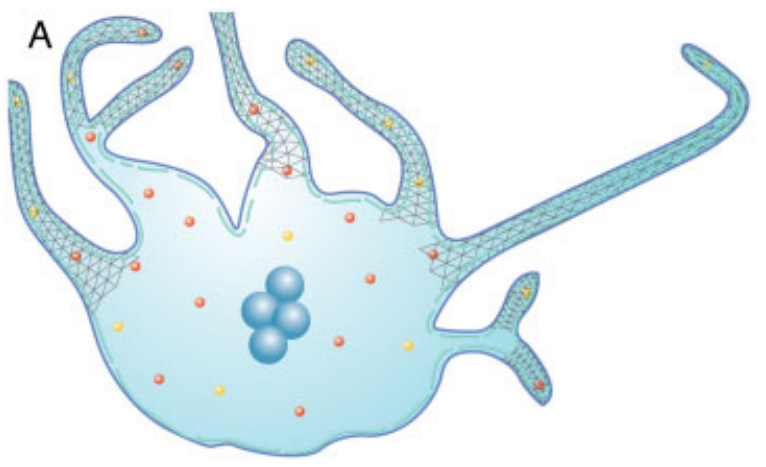

B

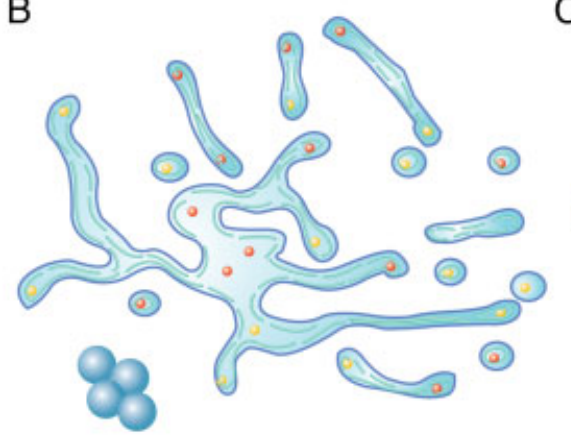

C

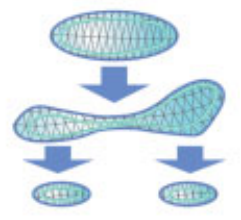

Fig. 3 Model of platelet production. (A) The formation of proplatelets begins with the extension of thick pseudopodia that use cortical bundles of microtubules to extend and form thin proplatelets with bulbous ends. Proplatelet membranes are laminated with an undercoat of spectrin. The ends of proplatelets contain a bundle of microtubules that loop on themselves. Proplatelet elongation involves the sliding of microtubules past one another, driven by the molecular motor cytoplasmic dynein. As proplatelets extend, development of the membrane surface area necessitates the outflow of the invaginated membrane reservoir, a process that requires reorganization of the membrane skeleton. Mitochondria and granules traffic (as indicated by the orange and yellow spheres) to the tips of proplatelets along microtubules, which function as the highways of the cell. Actin promotes the branching and amplification of proplatelet tips, representing a mechanism to augment the numbers of proplatelet tips and ultimately, platelets. (B) The entire megakaryocyte cytoplasm is converted into a mass of proplatelets. (C) Proplatelets continue to morph into preplatelets (anucleate discoid particles $2-10 \mu \mathrm{m}$ across), which are released from the cell. Preplatelets reversibly convert into barbell proplatelets (inset, top right), a process that is driven by microtubule-based forces. The membrane skeleton stabilizes this barbell form. Platelets release from proplatelet ends after the final fission event. The nucleus is eventually extruded from the proplatelets, ending the role of the megakaryocyte in this process.

microtubules are thickest in the region of the proplatelet near the body of the megakaryocyte but then thin to bundles of 5 to 10 microtubules near the tips of the proplatelets. The distal end of each proplatelet always has a platelet-size enlargement containing a microtubule bundle that makes a U-turn and loops just beneath the plasma membrane and reenters the shaft to form a teardrop-shaped or tennis racket-shaped structure. Because microtubule coils similar to those observed in blood platelets are detected only at the tips of proplatelets and not within the platelet-size swellings found along the length of proplatelets, putative platelets are generated only at the ends of proplatelets. ${ }^{18}$

By directly visualizing microtubule dynamics in living megakaryocytes using green fluorescent protein technology, we have obtained insights into how microtubules provide the

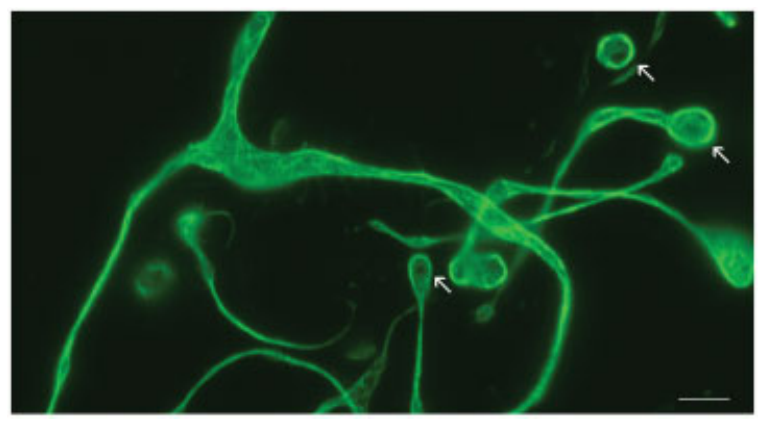

Fig. 4 Organization of microtubules within proplatelets. Immunofluorescence micrograph of murine megakaryocytes grown in culture and labeled with $\beta-1$ tubulin antibodies indicate that microtubules extend the entire length of proplatelets, including the tips and shaft. Immunofluorescence studies further demonstrate that coils (arrows) of microtubule similar to those seen in mature platelets occur in both proplatelets and released platelet-sized particles. Scale bar, $5 \mu \mathrm{m}$. force to power proplatelet elongation. ${ }^{20}$ End-binding protein three (EB3), a microtubule plus end-binding protein associated only with growing microtubules, attached to green fluorescent protein (GFP) was retrovirally expressed in mouse megakaryocytes and used as a marker to track the dynamics of microtubule plus ends. Round, immature megakaryocytes without proplatelets use a centrosomal-coupled microtubule nucleation/assembly reaction, which appears as a prominent starburst pattern when visualized with EB3-GFP. Microtubules assemble only from the centrosomes and grow outward into the cell cortex, where they turn and run in parallel with the cell edges. However, just before proplatelet production commences, centrosomal assembly ceases and microtubules begin to condense into the cortex. Fluorescence time-lapse microscopy of proplatelet-producing megakaryocytes expressing EB3-GFP demonstrates that as proplatelets extend, microtubule polymerization takes place constantly throughout the entire length of the proplatelet, including the tip, swellings, and shaft. The rates of microtubule assembly (average, $10.2 \mu \mathrm{m} / \mathrm{min}$ ) are about 10 -fold faster than the rate at which proplatelets grow, suggesting assembly and proplatelet elongation are not tightly coupled. ${ }^{20}$ The EB3-GFP studies also demonstrated that microtubules assemble in both directions in proplatelets. This reveals that the microtubules within the bundles have a mixed polarity (-Fig. 5).

Although microtubules are constantly assembling in proplatelets, polymerization by itself does not supply the force for proplatelet elongation. Proplatelets continue to extend at normal rates even when microtubule assembly is momentarily blocked by drugs that inhibit net microtubule polymerization, suggesting an alternative mechanism for proplatelet extension. In line with this idea, proplatelets have an internal microtubule sliding mechanism. ${ }^{20}$ The minus-end directed microtubule molecular motor protein cytoplasmic dynein 


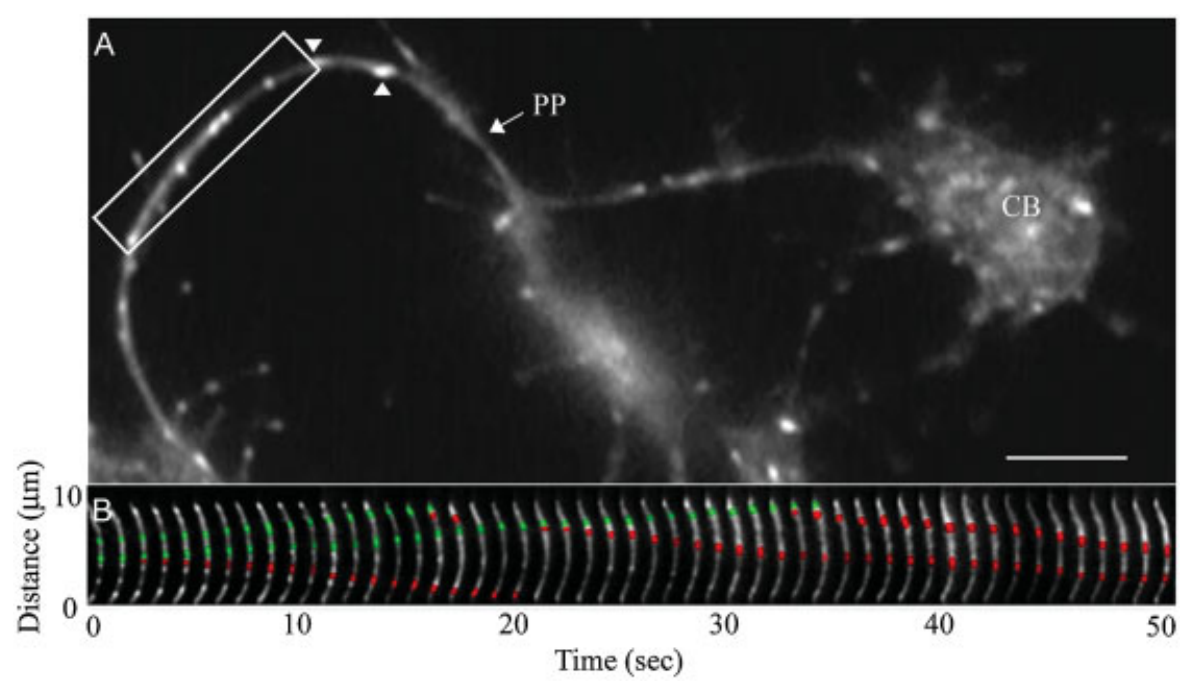

Fig. 5 Direct visualization of microtubule assembly in living megakaryocytes expressing end-binding protein three (EB3)-green fluorescent protein (GFP). (A) The first frame from a time-lapse movie of a living megakaryocyte that was directed to express EB3-GFP. The cell body (CB) is at the right of the micrograph and proplatelets (PP) extend to the left. EB3-GFP labels growing microtubule plus-ends in a characteristic "comet" staining pattern (arrowheads) that has a bright front and dim tail. The moving comets are found along the proplatelets as well as in the body of the megakaryocyte. Scale bar, 5 mm. (B) Kymograph (movement over time of the boxed region in A). Images are of every second. EB3-GFP comets undergo bidirectional movements in proplatelets demonstrating that microtubules are organized as bipolar arrays. Some EB3-GFP comets that move toward the tip are highlighted in green; others that move toward the cell body are highlighted in red.

localizes along the length of microtubules of the proplatelet. Cytoplasmic dynein appears to participate directly in microtubule sliding, because inhibition of cytoplasmic dynein, through dissociation of the dynactin complex, blocks proplatelet formation. Microtubule sliding can also be reactivated in permeabilized proplatelets. Addition of ATP, known to support the enzymatic activity of microtubule-based molecular motors, activates proplatelet elongation in the permeabilized proplatelets that contain both cytoplasmic dynein and its regulatory complex called dynactin. Thus, dynein-driven microtubule sliding appears to be a crucial event in powering proplatelet elongation.

\section{Amplification of Proplatelet Ends via Bending and Branching}

At the same time as the microtubule system is used to propel the extension of proplatelets, an actin-powered process is used to branch off the shaft of the proplatelet, in so doing increasing the number of proplatelet tips available to participate in platelet production ( $\mathbf{- F i g}$. 6). This unique process starts when a region of the proplatelet shaft is bent into a U-shape. A new daughter process next juts out from the middle of this bend and extends. Some of the microtubules within the bent segment of the loop separate from the bundle to form a bulge. Sliding and polymerization of these microtubules creates a new daughter proplatelet process. ${ }^{17}$ This branching mechanism is driven by actin-based forces and is inhibited when megakaryocytes are treated with drugs such as the cytochalasins, a family of toxins that block actin monomers from polymerizing. Although the precise details on how the actin cytoskeleton powers this event are lacking, actin filament assemblies occur periodically along the length of the proplatelet shaft and are used as muscles to bend the rigid micro- tubules. At these locations, actin filaments polymerize; although much is known about the regulation of actin polymerization in platelets, there is very little information on how actin polymerization is regulated or stimulated at these locations along proplatelets. Strong connections between the microtubules and the actin filaments must be established to transmit the actin-powered bending forces to the microtubule bundle. One possibility is that actin filament-associated myosin motors may provide the force for bending. Myosins comprise a family of molecular motor proteins most known for their role in the contraction of muscle and a wide range of other motilities. Certain diseases suggest an important role for myosin II in platelet generation. Mutations of MYH9 (the gene that encodes myosin IIA) that modify myosin IIA at the site involved in myosin thick filament formation are implicated in MYH9-related disorders (e.g., May-Hegglin anomaly, Sebastian syndrome), a disorder where platelet numbers are reduced in numbers and are abnormally large.

\section{Mechanisms of Organelle Transport and Capture during Platelet Production}

Essential in the process of platelet generation is the distribution of granules and organelles into nascent platelets. In addition to functioning as the primary machinery to elongate proplatelets, the microtubules that line the length of proplatelets provide a secondary role-highways for the transport and delivery of membrane, organelles, and granules into proplatelets and assembling platelets at the ends of proplatelets. By monitoring the distribution and dynamics of organelles/granules in living cells, it was established that individual organelles are sent one by one from the megakaryocyte body into the proplatelets, where they move in both directions until they are captured at the ends of proplatelets. ${ }^{21}$ 

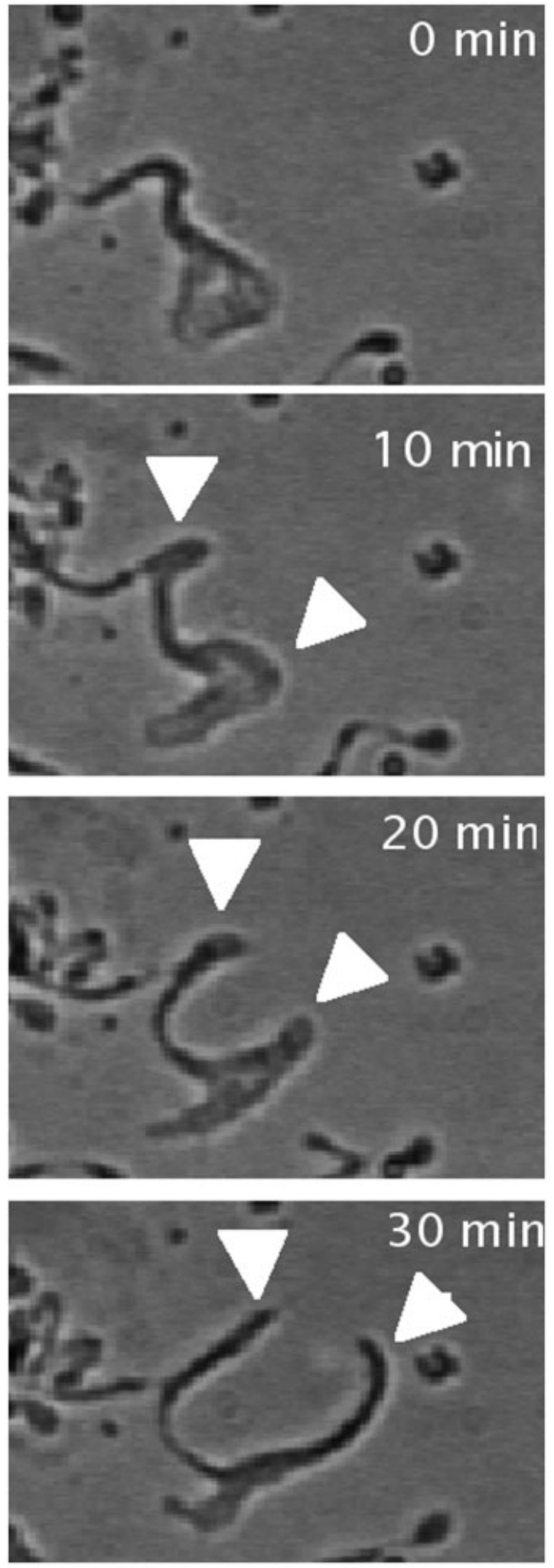

Fig. 6 Bending and branching of proplatelets. The bifurcation of proplatelets is observed in these phase-contrast images taken 10 minutes apart showing the bending and branching of a proplatelet extension. The bends are converted into loops that become compressed and extend, resulting in a bifurcation of the original tube. White arrowheads indicate the branch points. (Adapted from: Italiano JE Jr., Lecine P, Shivdasani RA, Hartwig JH. Blood platelets are assembled principally at the ends of proplatelet processes produced by differentiated megakaryocytes. J Cell Biol 1999;147(6):1299-1312.)
Thin-section electron microscopy and fluorescence microscopy studies indicate that organelles are in direct contact with microtubules, and actin inhibitors do not block the motion of organelles. Thus, motility appears to involve microtubulebased forces. Microtubules are polar structures with a clearly defined directionality, as indicated by a plus and minus end. The bidirectional movement of organelles is imparted by the bipolar organization of microtubules within the proplatelet. Beads coated with the molecular motor kinesin, which only moves in the plus-end direction, translocate in both directions over the microtubules within permeabilized proplatelets. Of the two major microtubule-based motors, only the plus-end-directed kinesin is located in a pattern similar to organelles and is most likely responsible for translocating these cargo along microtubules. ${ }^{21}$ Taken together, the data suggest that a twofold mechanism of organelle movement occurs during platelet production. First, organelles and granules move along microtubules, and, second, the microtubules themselves can slide in relation to other motile filaments to move organelles along proplatelets.

Spectrin-Based Membrane Skeleton in Platelet Production Although the role of microtubules and actin filaments in platelet production have been analyzed at length, the role of the membrane skeleton in this process has only recently emerged. Rapid-freeze high-resolution electron microscopy reveals that proplatelets contain a dense membrane skeleton similar in structure to that observed in the platelets in blood. $^{22}$ The main fibrous component of this skeleton is spectrin. The nonerythroid spectrin subunits, $\beta$-II and $\alpha$-II spectrin, are predominately expressed in megakaryocytes, proplatelets, and platelets. However, erythroid $\beta-\mathrm{I}$ and $\alpha-\mathrm{I}$ spectrin isoforms are also present. Assembly of spectrin tetramers is required for generation of the invaginated demarcation membrane system and ultimately proplatelet production because expression of a spectrin tetramer-disrupting peptide in megakaryocytes inhibits the progression of both. In addition, introduction of this spectrin-disrupting construct into a detergent-permeabilized model system rapidly destabilizes proplatelet morphology, resulting in enormous swelling and blebbing. Spectrin tetramers also stabilize the barbell shapes of the penultimate stage in platelet generation from proplatelets. $^{22}$ Taken together, these observations suggest a role for spectrin in distinct events of megakaryocyte development through its participation in the generation of demarcation membranes and in the maintenance of proplatelet structure.

\section{Release of Individual Platelets}

In vivo, proplatelets extend into bone marrow vascular sinusoids, where they are released and enter the bloodstream. Previously, our understanding of platelet release was based on static images of scanning electron micrographs of the bone marrow sinusoids. ${ }^{9}$ More recently, Junt and colleagues have used intravital fluorescence microscopy to directly visualize proplatelet production in the opened cranial marrow cavity of living mice. ${ }^{23}$ Fluorescently labeled megakaryocytes could be seen to protrude proplatelets and release megakaryocyte 
fragments into the marrow sinusoids of living mice. Notably, these anucleate mekaryocyte fragments typically exceed platelet dimensions, suggesting that platelet morphogenesis continues in the circulation. In line with these observations, we identified a previously unrecognized intermediate stage in platelet formation and release, which we termed the preplatelet. ${ }^{24}$ Preplatelets, which appear as "giant discoid platelets," 3 to $10 \mu \mathrm{m}$ in diameter, retain the capacity to convert into barbell-shaped proplatelets and undergo fission into individual platelets. Inhibitors of microtubule assembly block the transition of preplatelets to barbells. Furthermore, taxol, which stabilizes microtubules and stimulates microtubule polymerization, promotes the conversion of preplatelets into platelets. Thus, the conversion of preplatelets to barbell proplatelets is powered by microtubule-based forces. ${ }^{24}$ It is tempting to speculate that the preplatelet fission mechanism is a major determinant of platelet size and that some macrothrombocytopenias (Bernard-Soulier syndrome, MYH9-related disorders, etc.) represent a malfunction in converting preplatelets into barbell-shaped proplatelets. It is likely that the microtubule motors that drive proplatelet extension are involved in aspects of platelet release as well as in the process of microtubule coiling. Force constraints deriving from cortical microtubule band diameter and thickness play a major role in determining barbell conversion, and mathematical modeling suggests that platelet size is limited by microtubule bundling, elastic bending, and actin-myosin-spectrin cortical forces. In support of this concept, laser scanning cytometry has provided high-resolution images of both preplatelets and barbell-shapes in blood. ${ }^{25}$ It was demonstrated that individual human platelets have the innate capacity to duplicate and form new cell bodies that undergo fission into platelets. ${ }^{26}$ The morphologic similarities between platelets that form new cell bodies and preplatelets are striking. Whether or not newly released platelets exhibit a preplatelet phenotype, which may allow them to form barbell shapes and divide again, is not clear.

\section{Translating Thrombopoiesis Biology into Medicine}

It has been 17 years since the discovery of thrombopoietin, ${ }^{26}$ and drugs that are thrombopoietin mimetics are beginning to make an impact on the treatment of thrombocytopenia. Despite this progress, we need to address the next major advances in this field. In the United States alone more than 2 million platelet transfusions occur each year, all with platelets from volunteer donors. Because of their essential role in hemostasis, platelets are used for patients who have experienced traumatic injury or are undergoing chemotherapy. The supply of platelets has long been a problem for hospitals and blood banks. The short storage time (5 days) of platelet products, which must be kept at room temperature, is also a challenge. In addition, the risk of transfusion of transmitted diseases and shortages in supply provide additional problems associated with donor platelets. Clearly, the ability to continuously generate platelets ex vivo in a bioreactor would provide a more advanced way to generate a product to treat thrombocytopenia. Since thrombopoietin was identified as the major regulator of platelet production, it has been used to make enriched populations of megakaryocytes. In 1995 Choi and colleagues demonstrated that platelets generated in vitro from proplatelet-displaying human megakaryocytes were functional. ${ }^{11}$ Since then, both megakaryocytes and platelets have been differentiated from multiple sources, including embryonic stem cells and induced pluripotent stem cells. ${ }^{28,29}$ However, despite all these various sources of megakaryocytes and platelets, the yields of in vivo generated platelets have not come close to what is necessary for clinical application. In the bone marrow specialized microenvironments, called niches, regulate megakaryocyte development and platelet production through a complex crosstalk between many cell types. The establishment of an in vitro model that faithfully recapitulates the fundamental interactions of the niche components in a controlled setting could advance the development of in vitro platelets for transfusion. For example, Lasky and colleagues have constructed a purpose-built three-dimensional hydrogel scaffold that functions as a bioreactor for platelet production. In this system, the authors used CD34 positively selected human cord blood cells in a three-dimensional hydrogel scaffold coated with thrombopoietin and/or fibronectin to increase platelet output. ${ }^{30}$ In this three-dimensional model, the manipulation of oxygenation and flowinduced shear stress appears to increase the yield of in vitro platelets derived from cord blood. ${ }^{31}$ Building on these models, Pallotta and colleagues have also recently developed a silkbased three-dimensional system that partially recapitulates the spatial reconstruction of the bone marrow environment and produces platelets in vitro. ${ }^{32}$ Overall, these advances suggest that it will be important to mimic physiology and use biologically inspired engineering to advance this technology to the clinic.

\section{Platelets: Not Just the Band-Aids of the Blood}

Although their primary function is to prevent bleeding, recent data suggest that platelets contribute to a diverse array of processes that go way beyond thrombosis and hemostasis. Platelets have been implicated in many different processes, including the development of the lymphatic system, liver regeneration, inflammation, and cancer. ${ }^{33}$ From the perspective of platelet production, it will be important to understand how platelet generation is altered or "reprogrammed" to affect these other functions. We have recently investigated how platelets regulate angiogenesis and innate immunity.

\section{Platelets and New Blood Vessel Growth}

A body of clinical and experimental data suggest that platelets influence tumor development by transporting and delivering angiogenesis regulatory proteins. ${ }^{34,35}$ The ability of platelets to interact with the endothelium is a key factor that allows them to regulate angiogenesis. Some of the first data suggesting that platelets can modulate angiogenesis were reported by Gimbrone et al, who demonstrated that perfusion of plasma depleted of blood platelets resulted in instability of the endothelial layer and hemorrhages. ${ }^{36}$ Other studies in animals demonstrated that absence of platelets or low 
platelet count led to increased permeability of the vasculature, the likely result of excessive space between endothelial cells. It was later demonstrated that platelets modulate angiogenesis by showing that platelets could stimulate the formation of capillary-like and tubelike structures when added to human umbilical vein endothelial cells in culture. Although platelets have been presumed to contribute to tumor development by providing numerous stimulators and inhibitors of angiogenesis, the regulatory role of platelets in this process is not fully understood. Platelets contain numerous regulators of new blood vessel growth, which can be delivered to the endothelium when platelets activate. ${ }^{37}$ The proangiogenic regulatory proteins vascular endothelial growth factor, platelet-derived growth factor, epithelial growth factor, basic fibroblast growth factor, metalloproteinases, and sphingosine 1-phosphate have all been identified in platelets. ${ }^{38}$ The stimulators in platelets are counterbalanced by the platelet angiogenesis inhibitors, including platelet factor 4 , thrombospondin-1, endostatin, tissue inhibitor of matrix metalloproteinases, and angiostatin. ${ }^{38}$ Most angiogenesis regulatory proteins identified in platelets have been localized to $\alpha$-granules, the major storage granule of platelets. Each human platelet contains about 40 to $80 \alpha$ granules. By attaching to the endothelium of injured vessels and then releasing their contents, platelets deliver high concentrations of angiogenesis regulatory proteins in a precise manner.

The levels of these angiogenic regulatory proteins in platelets appear to play a role in tumor angiogenesis. It has been reported that when a microscopically sized human tumor is present in a mouse, circulating platelets take up and sequester specific angiogenesis regulatory proteins, such as platelet factor 4, vascular endothelial growth factor, and basic fibroblast growth factor. ${ }^{39}$ The angiogenic regulatory proteins are sequestered in the platelets at a significantly higher concentration than is observed in the plasma. This new platelet property may lead to the development of a biomarker for very early detection of tumor recurrence. ${ }^{40}$ Whereas an association between new blood vessel growth and platelets has long been recognized, the cause and effect relationship linking the two has been unclear. Given that platelets contain both stimulators and inhibitors of angiogenesis, packaged into a homogeneous population of $\alpha$-granules, the question becomes how can platelets have either a proangiogenic or antiangiogenic effect? The release of a mixture of both proand antiangiogenic regulatory proteins from platelets should cancel the effect of each other. Several groups have demonstrated that platelets can preferentially secrete a platelet releasate that has either a pro- or antiangiogenic effect. ${ }^{41,42}$ The treatment of human platelets with the selective protease activated receptor (PAR)-4 agonist resulted in release of endostatin-containing granules but not vascular endothelial growth factor-containing granules, whereas the selective PAR-1 agonist liberated vascular endothelial growth factor but not endostatin-containing granules. This differential release is also observed with physiologic agonists. ${ }^{43}$ Activation of human platelets with adenosine diphosphate stimulates the release of vascular endothelial growth factor but not endostatin, whereas thromboxane $\mathrm{A} 2$ releases endostatin but not vascular endothelial growth factor. Activation with adenosine diphosphate also promotes the formation of capillary structures by human umbilical vein endothelial cells in vitro. Conversely, thromboxane A2-activated releasate inhibits formation of capillary structures. We have also tested the hypothesis that cancer cells preferentially stimulate platelets to secrete their proangiogenic payload, providing a mechanism for how tumors may hijack platelets to promote new blood vessel growth. In support of this idea, the MCF-7 breast cancer cell line stimulates secretion of vascular endothelial growth factor and a proangiogenic releasate from platelets. Interestingly, the antiplatelet agent aspirin blocked plateletmediated angiogenesis after introduction to the MCF-7 cells, pointing to a potential mechanism for how aspirin may influence malignancy. ${ }^{43}$ Taken together, these data suggest that manipulation of differentially mediated release of angiogenesis regulatory proteins from platelets may provide a new modality for treatment of cancer. A better understanding of the mechanisms by which platelets release angiogenic regulatory proteins should yield strategies for therapeutic benefit.

\section{Platelets and Immunity}

Platelets play a role in immunity by expressing members of the toll-like receptor (TLR) family. ${ }^{44}$ TLRs are a class of proteins that play an essential role in the innate immune system by recognizing molecules that are broadly shared by pathogens. TLRs have been extensively characterized in macrophages, dendritic cells, and neutrophils and support immune activation in response to conserved molecular motifs on pathogens. Although platelets express TLRs 1 through 9, most work has focused on TLRs 1 to 6 , which are expressed on the surface of the platelet and believed to trap bacteria for elimination by phagocytes. ${ }^{45-47}$ We recently demonstrated that the TLR9 transcript is specifically up-regulated during platelet production and is localized to a novel electron-dense tubular system named the T-granule. ${ }^{48}$ TLR9 colocalizes with protein disulfide isomerase and is associated with either vesicle-associated membrane protein 8 or vesicle-associated membrane protein 7 , which are molecules involved in vesicle fusion that regulate TLR9 distribution in platelets during activation. Type IV collagen specifically increases P-selectin, a cell adhesion molecule, and TLR9 surface expression and augments oligodeoxynucleotide sequestration and platelet aggregation upon the addition of viral and bacterial oligodeoxyribonucleotides. ${ }^{48}$ Increased surface expression of TLR9 in platelets and type $\mathrm{C} \mathrm{CpG} \mathrm{sequestration} \mathrm{may} \mathrm{aid} \mathrm{in} \mathrm{the}$ regulation and sequestration of circulating levels of bacterial DNA in the blood and consequently help manage the inflammatory response after lysis of bacterial cells. A more thorough understanding of the function that TLRs play in regulating the activation of platelets will likely yield new therapies for the treatment of cardiovascular infections.

\section{Conclusions}

The transition from megakaryocyte to released platelets is an elaborate and complex process. Although the basic 
mechanisms of platelet production have been investigated, elucidating the specific molecular controls and cellular events involved in platelet formation and release is an important goal. Among the major questions regarding platelet biogenesis that have yet to be addressed include the following: (1) What factors induce proplatelet formation in mature megakaryocytes? (2) How can we mimic human physiology to generate a bioreactor that produces in vitro platelets? (3) What are the regulatory signals that direct the sequence of developmental events that drives proplatelet production? (4) How can we engineer designer platelets that will deliver biologically active molecules? (5) What mechanisms contribute to the giant platelets observed in macrothrombocytopenias? Further examination of the molecular, cellular, and biochemical studies of megakaryocytes as they transition into platelets will provide a clearer understanding of these processes. This knowledge may lead to the ex vivo generation of platelets or in vivo therapies aimed at enhancing platelet production in patients with thrombocytopenia.

\section{References}

1 Hartwig JH, DeSisto M. The cytoskeleton of the resting human blood platelet: structure of the membrane skeleton and its attachment to actin filaments. J Cell Biol 1991;112(3):407-425

2 White JG. Effects of colchicine and vinca alkaloids on human platelets. I. Influence on platelet microtubules and contractile function. Am J Pathol 1968;53(2):281-291

3 Schwer HD, Lecine P, Tiwari S, Italiano JE Jr, Hartwig JH, Shivdasani RA. A lineage-restricted and divergent beta-tubulin isoform is essential for the biogenesis, structure and function of blood platelets. Curr Biol 2001;11(8):579-586

4 Nakamura F, Pudas R, Heikkinen O, et al. The structure of the GPIbfilamin A complex. Blood 2006;107(5):1925-1932

5 Andrews RK, Fox JE. Identification of a region in the cytoplasmic domain of the platelet membrane glycoprotein Ib-IX complex that binds to purified actin-binding protein. J Biol Chem 1992; 267(26):18605-18611

6 López JA, Andrews RK, Afshar-Kharghan V, Berndt MC. BernardSoulier syndrome. Blood 1998;91(12):4397-4418

7 Ware J, Russell S, Ruggeri ZM. Generation and rescue of a murine model of platelet dysfunction: the Bernard-Soulier syndrome. Proc Natl Acad Sci USA 2000;97(6):2803-2808

8 Kaushansky K, Drachman JG. The molecular and cellular biology of thrombopoietin: the primary regulator of platelet production. Oncogene 2002;21(21):3359-3367

9 Becker RP, De Bruyn PP. The transmural passage of blood cells into myeloid sinusoids and the entry of platelets into the sinusoidal circulation; a scanning electron microscopic investigation. Am J Anat 1976;145(2):183-205

10 Radley J. Ultrastructural aspects of platelet production. Megakaryocyte development and function. Prog Clin Biol Res 1986;215: 387-398

11 Choi ES, Nichol JL, Hokom MM, Hornkohl AC, Hunt P. Platelets generated in vitro from proplatelet-displaying human megakaryocytes are functional. Blood 1995;85(2):402-413

12 Shivdasani RA, Rosenblatt MF, Zucker-Franklin D, et al. Transcription factor NF-E2 is required for platelet formation independent of the actions of thrombopoietin/MGDF in megakaryocyte development. Cell 1995;81(5):695-704

13 Zucker-Franklin D, Petursson S. Thrombocytopoiesis-analysis by membrane tracer and freeze-fracture studies on fresh human and cultured mouse megakaryocytes. J Cell Biol 1984;99(2):390-402
14 Radley JM, Haller CJ. The demarcation membrane system of the megakaryocyte: a misnomer? Blood 1982;60(1):213-219

15 Schulze H, Korpal M, Hurov J, et al. Characterization of the megakaryocyte demarcation membrane system and its role in thrombopoiesis. Blood 2006;107(10):3868-3875

16 Yamada E. The fine structure of the megakaryocyte in the mouse spleen. Acta Anat (Basel) 1957;29(3):267-290

17 Cramer EM, Norol F, Guichard J, et al. Ultrastructure of platelet formation by human megakaryocytes cultured with the $\mathrm{Mpl}$ ligand. Blood 1997;89(7):2336-2346

18 Italiano JE Jr, Lecine P, Shivdasani RA, Hartwig JH. Blood platelets are assembled principally at the ends of proplatelet processes produced by differentiated megakaryocytes. J Cell Biol 1999;147 (6):1299-1312

19 Tablin F, Castro M, Leven RM. Blood platelet formation in vitro. The role of the cytoskeleton in megakaryocyte fragmentation. J Cell Sci 1990;97(Pt 1):59-70

20 Patel SR, Richardson JL, Schulze H, et al. Differential roles of microtubule assembly and sliding in proplatelet formation by megakaryocytes. Blood 2005;106(13):4076-4085

21 Richardson JL, Shivdasani RA, Boers C, Hartwig JH, Italiano JE Jr. Mechanisms of organelle transport and capture along proplatelets during platelet production. Blood 2005;106(13):4066-4075

22 Patel-Hett S, Wang H, Begonja AJ, et al. The spectrin-based membrane skeleton stabilizes mouse megakaryocyte membrane systems and is essential for proplatelet and platelet formation. Blood 2011;118(6):1641-1652

23 Junt T, Schulze H, Chen Z, et al. Dynamic visualization of thrombopoiesis within bone marrow. Science 2007;317(5845):1767-1770

24 Thon JN, Montalvo A, Patel-Hett S, et al. Cytoskeletal mechanics of proplatelet maturation and platelet release. J Cell Biol 2010;191 (4):861-874

25 Thon JN, Macleod H, Begonja AJ, et al. Microtubule and cortical forces determine platelet size during vascular platelet production. Nat Commun 2012;3:852

26 Schwertz H, Köster S, Kahr WH, et al. Anucleate platelets generate progeny. Blood 2010;115(18):3801-3809

27 Kaushansky K, Lok S, Holly RD, et al. Promotion of megakaryocyte progenitor expansion and differentiation by the c-Mpl ligand thrombopoietin. Nature 1994;369(6481):568-571

28 Lu SJ, Li F, Yin H, et al. Platelets generated from human embryonic stem cells are functional in vitro and in the microcirculation of living mice. Cell Res 2011;21(3):530-545

29 Takayama N, Nishimura S, Nakamura S, et al. Transient activation of c-MYC expression is critical for efficient platelet generation from human induced pluripotent stem cells. J Exp Med 2010;207 (13):2817-2830

30 Sullenbarger B, Bahng JH, Gruner R, Kotov N, Lasky LC. Prolonged continuous in vitro human platelet production using three-dimensional scaffolds. Exp Hematol 2009;37(1):101-110

31 Lasky LC, Sullenbarger B. Manipulation of oxygenation and flowinduced shear stress can increase the in vitro yield of platelets from cord blood. Tissue Eng Part C Methods 2011;17(11):1081-1088

32 Pallotta I, Lovett M, Kaplan DL, Balduini A. Three-dimensional system for the in vitro study of megakaryocytes and functional platelet production using silk-based vascular tubes. Tissue Eng Part C Methods 2011;17(12):1223-1232

33 Leslie M. Cell biology. Beyond clotting: the powers of platelets. Science 2010;328(5978):562-564

34 Pinedo HM, Verheul HM, D’Amato RJ, Folkman J. Involvement of platelets in tumour angiogenesis? Lancet 1998;352(9142): 1775-1777

35 Verheul HM, Hoekman K, Luykx-de Bakker S, et al. Platelet: transporter of vascular endothelial growth factor. Clin Cancer Res 1997;3(12 Pt 1):2187-2190

36 Gimbrone MA Jr, Aster RH, Cotran RS, Corkery J, Jandl JH, Folkman J. Preservation of vascular integrity in organs perfused in vitro with a platelet-rich medium. Nature 1969;222(5188):33-36 
37 Möhle R, Green D, Moore MA, Nachman RL, Rafii S. Constitutive production and thrombin-induced release of vascular endothelial growth factor by human megakaryocytes and platelets. Proc Natl Acad Sci USA 1997;94(2):663-668

38 Browder T, Folkman J, Pirie-Shepherd S. The hemostatic system as a regulator of angiogenesis. J Biol Chem 2000;275(3): 1521-1524

39 Klement GL, Yip TT, Cassiola F, et al. Platelets actively sequester angiogenesis regulators. Blood 2009;113(12):2835-2842

40 Cervi D, Yip TT, Bhattacharya N, et al. Platelet-associated PF-4 as a biomarker of early tumor growth. Blood 2008;111(3): 1201-1207

41 Italiano JE Jr, Richardson JL, Patel-Hett S, et al. Angiogenesis is regulated by a novel mechanism: pro- and antiangiogenic proteins are organized into separate platelet alpha granules and differentially released. Blood 2008;111(3):1227-1233

42 Ma L, Perini R, McKnight W, et al. Proteinase-activated receptors 1 and 4 counter-regulate endostatin and VEGF release from human platelets. Proc Natl Acad Sci USA 2005;102(1):216-220
43 Battinelli EM, Markens BA, Italiano JE Jr. Release of angiogenesis regulatory proteins from platelet alpha granules: modulation of physiologic and pathologic angiogenesis. Blood 2011;118 (5):1359-1369

44 Semple JW, Italiano JE Jr, Freedman J. Platelets and the immune continuum. Nat Rev Immunol 2011;11(4):264-274

45 Andonegui G, Kerfoot SM, McNagny K, Ebbert KV, Patel KD, Kubes P. Platelets express functional toll-like receptor-4. Blood 2005; 106(7):2417-2423

46 Aslam R, Speck ER, Kim M, et al. Platelet Toll-like receptor expression modulates lipopolysaccharide-induced thrombocytopenia and tumor necrosis factor-alpha production in vivo. Blood 2006;107(2):637-641

47 Clark SR, Ma AC, Tavener SA, et al. Platelet TLR4 activates neutrophil extracellular traps to ensnare bacteria in septic blood. Nat Med 2007;13(4):463-469

48 Thon JN, Peters CG, Machlus KR, et al. T granules in human platelets function in TLR9 organization and signaling. J Cell Biol 2012; 198(4):561-574 